\title{
New trends in translational microsurgery ${ }^{1}$
}

Eiji Kobayashi'

'MD, PhD, Department of Organ Fabrication, Keio University School of Medicine, Tokyo, Japan. Conception and design of the study, manuscript writing, critical revision.

\section{Abstract}

Technological advances such as optical instruments and surgical tools have enabled the considerable contributions of microsurgery to surgical therapies. Accordingly, surgical therapeutics has provided the latest information across a wide range of medical specialties, including immunology and pharmacology, despite specialization according to organs and organ systems. The International Society for Experimental Microsurgery, an academic organization, has utilized experimental microsurgery technology in the identification of curative concepts for diseases that remain difficult to treat. For this publication to mark the 32nd anniversary of the Brazilian Surgical Society, I introduced the following types of technology related to the further development of microsurgical technological innovations in the future: high-resolution three-dimensional (3D) video and touch-sensitive microsurgery robots.

Key words: Microsurgery. Video-Assisted Surgery. Robotics.

\section{- Introduction}

Microsurgery is a precise type of surgical operation in which tools such as loupes and microscopes are used when performing surgical procedures. In the latter half of the 18th century, advances in optical lens technology led to the development of magnifying instruments such as loupes. Stationary microscopes were introduced in clinical settings in the 19th century. The use of this technology expanded into the following medical fields: ear, nose and throat (ENT), plastic surgery, ophthalmology and neurosurgery ${ }^{1}$. Concurrently, a groundbreaking type of surgical treatment was introduced. Specifically, organ transplantation therapy was introduced with the aim of saving lives by transplanting new organs into patients who had experienced lethal organ failure ${ }^{2}$. Brazil was a leading clinical center worldwide during the early stages of transplantation therapy; here, pioneers in the field were performing organ transplantation in cases that were otherwise difficult to treat $^{3}$. However, it was difficult to achieve good clinical results with transplantation, and better immunosuppressive and organ preservation methods were urgently needed. In the latter half of the 19th century, highly specific organ transplantation research involving large numbers of laboratory animals was conducted to enable the transition from concept to reality. However, it was necessary to introduce excellent microsurgery technologies, especially for the creation of small animal 
models that were similar to humans. Dr. Sun Lee, the founder of the International Society for Experimental Microsurgery (ISEM), was the first person to develop a rat organ transplant model and disseminate that technology worldwide ${ }^{4}$.

However, it remained necessary to overcome the difficulties encountered when integrating medical specialties based on organs and organ systems and thus use an interdisciplinary approach that combined immunology, pharmacology and physiology. In 1992, Dr. Lee started the ISEM as an organization that aimed to take a more interdisciplinary approach and address all steps in the research process, from foundational research to clinical application. In 2010, ISEM held its $10^{\text {th }}$ conference in Sao Paulo, Brazil, with Professor Edna Montero serving as the chairman. At that time, I delivered a lecture regarding my views on the type of microsurgery that encompasses the spectrum from foundational research to clinical application, according to the future aims of ISEM, and discussed renaming "Microsurgery" as "Translational Microsurgery" with the intent to promote the creation of new treatments ${ }^{5}$.

Currently, revolutionary progress in the field of medical optical instruments is ongoing, and surgical techniques are beginning to incorporate robotic technology. This year, as the Brazilian Journal of Surgery celebrates its $32^{\text {nd }}$ birthday, we would like to introduce two new types of technology that we hope will have great impacts on the world of microsurgery.

\section{Changes in the International Society for Experimental Microsurgery (ISEM)}

Table 1 shows the locations of all previous international conferences, as well as the respective chairmen. In 2010, 26 years after the founding of this organization, the 10th ISEM international conference was held in Sao Paulo, Brazil. At that conference, Professor Saul Goldenberg served as the honorary organizer, Professor Edna Montero and Professor Murched O. Taha served as co-hosts, and I served as the 10th ISEM chairman (Figure 1).

Table 1 - The year and place in which each conference was held, as well as the names of the chairman of each conference.

International Society for Experimental Microsurgery

\begin{tabular}{ll}
\hline 1992 1st. ISEM Rome, Italy & Masumi Nozawa (Japan) \\
1994 2nd. ISEM Kanazawa, Japan & Amulf Thiede (Germany) \\
1996 3rd. ISEM Wuerzburg, Germany & Giuseppe Mazzoni (USA) \\
1998 4th. ISEM London/Ontario, Canada & Teruho Kajimoto (Japan) \\
2000 5th. ISEM Catania, Italy & Robert Zhong (Canada) \\
2002 6th. ISEM San Diego, USA & Antonio Di Cataldo (Italy) \\
2004 7th. ISEM Debrecen, Hungary & Shiguang Qian (USA) \\
2006 8th. ISEM Montreal, Canada & Heinz-Jochen Gassel (Germany) \\
2008 9th. ISEM Shanghai, People's Republic of China & Corradino Campisi (Italy) \\
2010 10th. ISEM Sao Paulo, Brazil & Eiji Kobayashi (Japan) \\
2012 11th. ISEM Timisoara, Romania & Edna F.S. Montero (Brazil) \\
2014 12th. ISEM Kyoto, Japan & Huifang Chen, MD (Canada) \\
2016 13th. ISEM Tianjin, China & Norbert Nemeth (Hungary) \\
2018 14th. ISEM Debrecen, Hungary & Mihai Ionac (Romania) \\
\hline
\end{tabular}




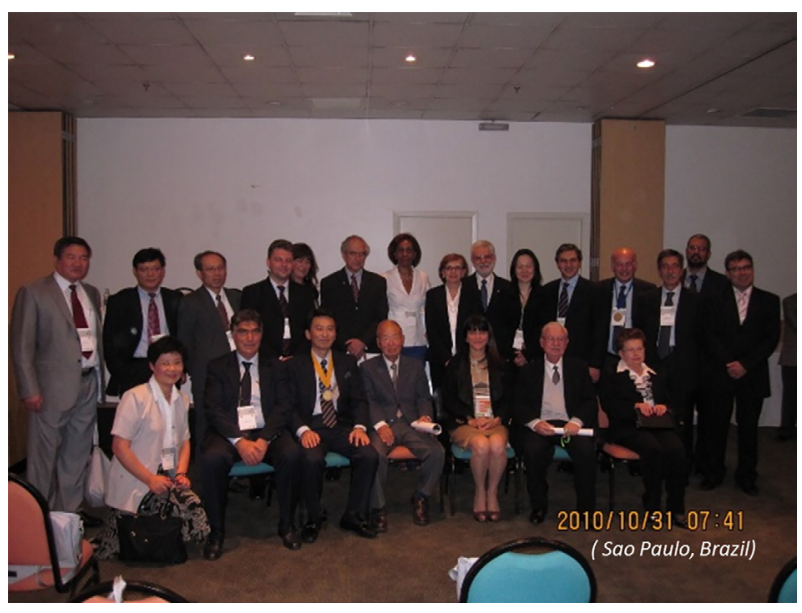

Figure 1 - Group photo of the ISEM board members in Sao Paulo, Brazil in 2010.

These ISEM academic conferences are held every 2 years, and topics such as foundational research and cutting edge clinical achievements, as well as microsurgery-related education, are routinely discussed. In the next section, I will discuss two types of microsurgery technology on which I have recently worked.

\section{Application of high-resolution 3D video microscopes to microsurgery}

Microsurgery with a conventional optical microscope provides a limited field of view and imposes serious strain on surgeons during long operations. Accordingly, an ophthalmological surgical technique known as "heads-up surgery" was recently developed; here, the field of view is displayed on a screen via a high-resolution camera ${ }^{6}$. This method is now being applied to various types of microsurgeries with long operating times that formerly required the use of a magnifying glass. Heads-up surgery could be implemented in an even greater range of operations. Additionally, many microsurgeries could implement headsup surgery with a three-dimensional (3D) video camera. Currently available higherperformance CCD cameras and imaging technologies will enable new options for advanced microsurgery.

In our specialty, arterial reconstruction for living-donor pediatric liver transplantation, microsurgery has the following characteristics ${ }^{5,7}$ :

1. Narrow field of view;

2. Deep operating field ( 1 and 2 also apply to brain surgery);

3. Respiratory movement (variable focal depth);

4. Requirement for the use of a microscope (microscopy setup: 15-30 minutes).

To overcome these issues, the following improvements can be made using the headsup method with a 3D-4K video system.

1. The camera is mounted in the center of the surgical light, and the zoom function is used to adapt the camera to a wide variety of surgical procedures (Figure 2);

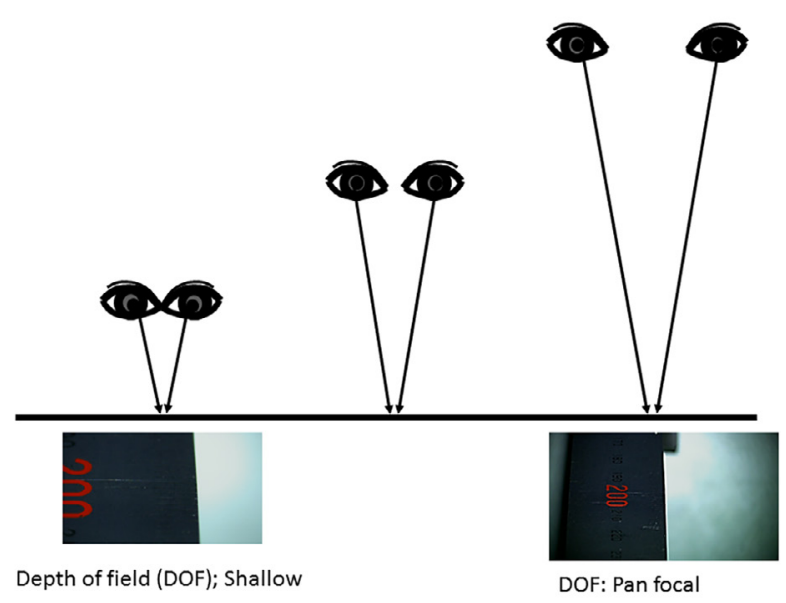

Figure 2 - Three-dimensional conceptual diagram of details viewed up close and at a distance. When magnified at a distance, a pan-focus view is obtained as the depth of field increases.

2. The operator and assistant have the same field of view during heads-up surgery (Figure 3); 


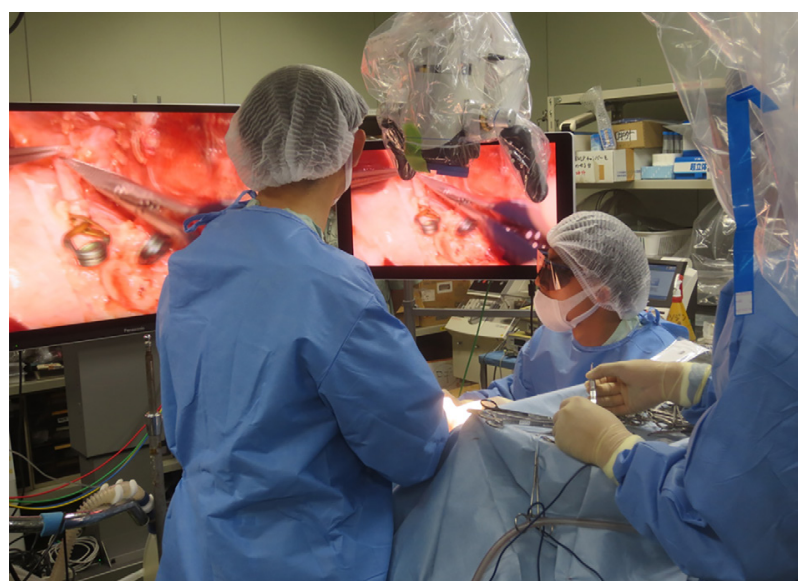

Figure 3 - Testing of the latest three-dimensional (3D)-4K videoscope on a porcine model.

The photo depicts surgeons performing a microsurgery operation (seated) and their assistants (standing). Both groups can look directly at the 3D image (Photo: Kyoto University School of Medicine, Animal Experimentation Laboratory).

3. A 3D view of the operating field is available. Accordingly, a dualeye (two HD sensors) system will provide greater evolutional views;

4. Given the viewing distance, the focal depth becomes pan-focal and is not greatly disturbed by pulsing blood vessels or movement (e.g., respiratory);

5. The video system can be used in conjunction with fluorescence imaging, such as indocyanine green, to measure blood flow and identify malignancies.

We hope that this new 3D-4K video system will reduce surgical complications and increase patient safety. This system should simultaneously relieve the stress and neck pain experienced by operators.

\section{- Development of robot-assisted microsurgical forceps with haptic feedback}

Today, many surgical procedures are performed arthroscopically. Surgical techniques are often practiced using dummies, cadavers and live pigs (Figure 4).

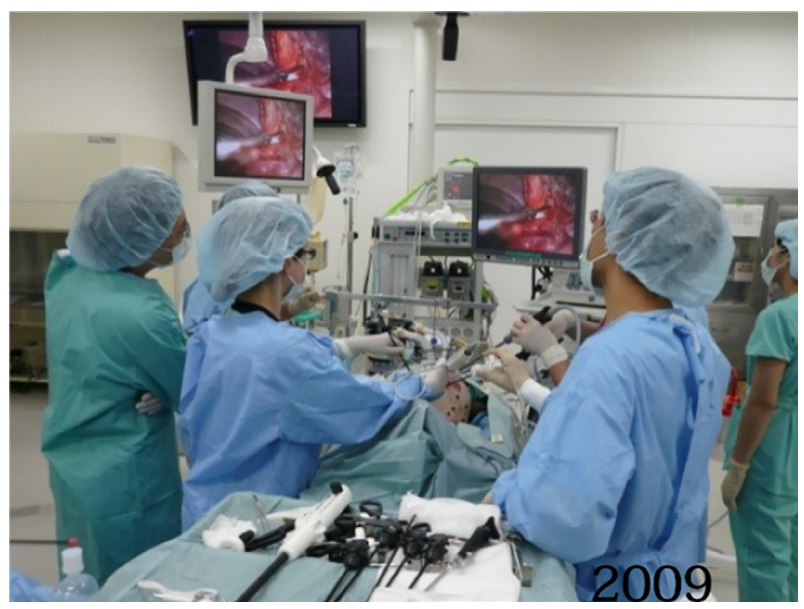

Figure 4 - Arthroscopic surgery training using a porcine model. This type of surgical practice has been common since the early 2000s. In recent years, it has also been used for robotic surgery training (Photo: Jichi Medical University CDAMTec).

In recent years, the development of robot-assisted surgery has led to an eminent revolution in surgical operations. In the field of microsurgery, the world's first supermicrosurgery operation was performed using Microsure, or "robot hands," at Maastricht University Medical Center+ in 2017. However, robots may experience difficulties when interacting with uncertain environments. Optical technology, while enhancing the scope of a surgeon's eyesight, decreases the haptic sensation in the surgeon's hands. In microsurgery, the haptic sensation from extremely thin suture threads is nearly undetectable, and expert surgeons tend to recoup this lack of haptic sensation from microscopic images. Similar to microscopes and other engineering supports for eyes, surgical robots have been developed to support hands. Nevertheless, these robots cannot yet transmit haptic information. Here, I would like to introduce recent advances in robot-assisted microsurgery that include the use of haptic 
technology ${ }^{8}$.

Bilateral control, an engineering methodology used to transmit haptic sensation, has been developed via senseless technology (Figure 5$)^{9}$. With this method, haptics can be controlled mutually between the "Master," which is operated by a human, and the "Slave," which directly manages surgical sites. We have further extended our research to the topic of "scaling bilateral control," wherein the haptic force is magnified between the "Master" and "Slave". A novel device equipped with scaling bilateral control system has been partially developed ${ }^{9}$. An attempt was made to prove the ability of a magnifying tensile force to identify the extremely thin threads used in microsurgery. We successfully demonstrated the ability to pull a thread of spider silk (Figure 6).
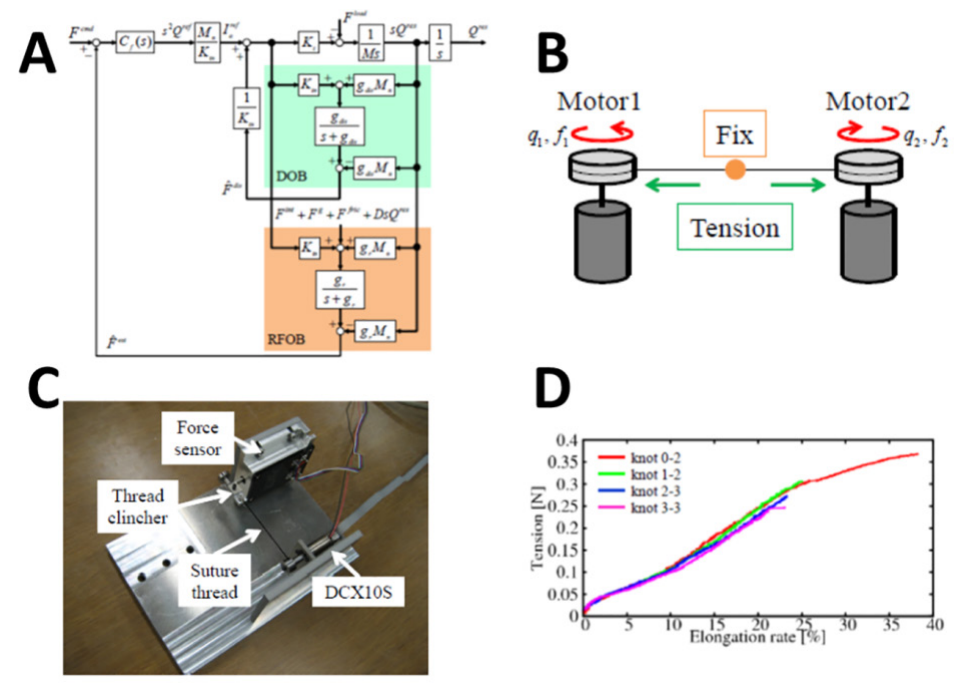

Figure 5 - The haptic system developed by the Faculty of Science and Technology at Keio University. A. Overview diagram; B. Simple micro-threader; C. Actual micro-threader; D. Micro-threading results (from reference 9).

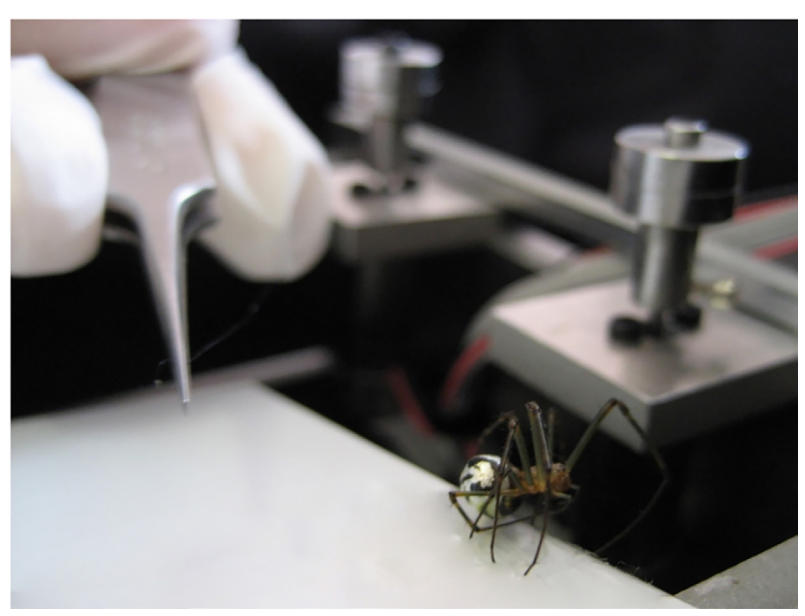

Figure 6 - Use of tweezers to pull a thread of spider silk. Although the spider is artificial, this image demonstrates haptic technology can be used to draw an item as thin as a strand of spider silk, without severing the strand.
In recent years, "soft robotics" research, which involves robots composed of soft materials, has aimed to increase adaptability in such environments. Similarly, we have aimed to use bilateral control to solve the existing difficulties. Bilateral control enables us to operate a robot with haptic sense feedback. The robots can act "softly" via the bilateral control system, despite being composed of steel or aluminum. A surgical robot is one promising application of this technology.

\section{- References}

1. Tamai S. History of microsurgery from the beginning until the end of the 1970s.

2. Microsurgery. 1993;14(1):6-13. PMID: 
8441345.

3. Murray JE. The Nobel Lectures in Immunology. The Nobel Prize for Physiology or Medicine, 1990. The first successful organ transplants in man. Scand J Immunol. 1994;39(1):1-11. PMID: 8290887.

4. Okumura $M$, Mester $M$. The coming of age of small bowel transplantation: a historical perspective. Transplant Proc. 1992;24(3):1241-2. PMID: 1604601.

5. Nemeth N. Tribute to the "Father" of experimental microsurgery, Professor Sun Lee (1920-2015). Microsurgery. 2016;36(2):97-8. doi: 10.1002/micr.30015.

6. Kobayashi E, Haga J. Translational microsurgery. A new platform for transplantation research. Acta Cir Bras. 2016;31(3):212-7. doi: 10.1590/S0102865020160030000010.

7. Eckardt C, Paulo EB. Heads-up surgery for vitreoretinal procedures: an experimental and clinical study. Retina. 2016;36(1):13747. doi: 10.1097/IAE.0000000000000689.

8. Sanada Y, Wakiya T, Hishikawa S, Hirata Y, Yamada N, Okada N, Ihara Y, Urahashi T, Mizuta K, Kobayashi E. Risk factors and treatments for hepatic arterial complications in pediatric living donor liver transplantation. J Hepatobiliary Pancreat Sci. 2014;21(7):463-72. doi: 10.1002/jhbp.

9. Deshpande N, Chauhan M, Pacchierotti C, Prattichizzo D, Caldwell DG, Mattos LS. Robot-assisted microsurgical forceps with haptic feedback for transoral laser microsurgery. Conf Proc IEEE Eng Med Biol Soc. 2016;2016:5156-9. doi: 10.1109/ EMBC.2016.7591888.

10.Oda K, Ohnishi K, Kobayashi E. Verification of a developed testing machine for suture threads through measurement of tension and elongation. 14th International Workshop on Advanced Motion Control, AMC 2016. Institute of Electrical and Electronics Engineers Inc; 2016. p.186-91. doi: 10.1109/AMC.2016.7496348.

\section{Acknowledgements}

The 3D-4K project has received support from the Japanese government (AMED) for a 3-year period (2017-2019), in collaboration with Mitaka Kohki and Panasonic Ltd. in the industrial sector and the Faculty of Medicine at Kyoto University (Dr. Shintaro Yagi and Professor Shinji Uemoto) and the Keio University School of Medicine in the academic sector.

This project (principal investigator: Kawana DDS, Keio University School of Medicine) is also supported by the Japanese government (AMED) for a 3-year period (20172019), in collaboration with the Department of System Design Engineering, Keio University (Professors Takahiro Nozaki and. Kouhei Ohnishi).

\section{Correspondence:}

Eiji Kobayashi

Department of Organ Fabrication, Keio University School of Medicine

35 Shinanomachi, Shinjuku-ku, Tokyo 160-8582 Japan

Phone: 81-3-5315-4090

organfabri@keio.jp

Received: May 08, 2018

Review: July 05, 2018

Accepted: Aug 03, 2018
Conflict of interest: none

Financial source: none 